\title{
CARACTERIZAÇÕES ANATÔMICA E FÍSICO-MECÂNICA DA MADEIRA DE Liquidambar sp.
}

\author{
Thaís Pereira Freitas ${ }^{1}$, Lairo David Feuchard ${ }^{1}$, José Tarcísio Silva Oliveira ${ }^{2}$, Juarez Benigno Paes ${ }^{2 *}$, \\ Marina Donária Chaves Arantes ${ }^{2}$ \\ ${ }^{1}$ Programa de Pós Graduação em Ciências Florestais, Universidade Federal do Espírito Santo, Jerônimo Monteiro - ES, Brasil - \\ thais_pfreitas@yahoo.com.br; lairodavid@hotmail.com \\ ${ }^{2}$ Departamento de Ciências Florestais e da Madeira, Universidade Federal do Espírito Santo, Jerônimo Monteiro - ES, Brasil - \\ jtsilva@npd.ufes.br; juarez.benigno@pq.cnpq.br; marina@cca.ufes.br
}

Recebido para publicação: 20/06/2014 - Aceito para publicação: 24/06/2015

\begin{abstract}
Resumo
Este trabalho teve como objetivo a caracterização anatômica e físico-mecânica da madeira de Liquidambar sp. Para tanto, foram utilizadas três árvores com oito anos de idade, provenientes de um arboreto localizado do município de Guaçuí, Espírito Santo. As características anatômicas analisadas foram as dimensões das fibras (comprimento, diâmetro total e do lume e espessura da parede), dos elementos de vasos (diâmetro tangencial e frequência) e dos raios (altura, largura e frequência). Para a caracterização físico-mecânica foram avaliadas a massa específica (básica, aparente a 12\% de umidade e anidra), as contrações totais lineares e volumétrica, a resistência à flexão estática, à compressão paralela às fibras, ao cisalhamento e dureza Janka. Constatou-se frequência vascular numerosa com vasos de pequeno diâmetro tangencial, raios heterocelulares ocorrendo em baixa frequência, e fibras libriformes longas de paredes delgadas a espessas. A massa específica básica da madeira proveniente de discos retirados a $1,30 \mathrm{~m}$ do nível do solo foi de $0,48 \mathrm{~g} \mathrm{~cm}^{-3}$, sendo considerada média, a estabilidade dimensional foi considerada baixa, e houve grande influência da umidade nos valores de resistência da madeira.
\end{abstract}

Palavra-chave: Anatomia; massa específica; estabilidade dimensional; resistência mecânica.

\begin{abstract}
Anatomical and physical-mechanical characterization of Liquidambar sp. wood. This research aimed to improve anatomical, physical and mechanical characterization of Liquidambar sp.wood. Three trees were obtained, at eight years of age, from an arboretum located in Guaçuí, Espírito Santo State, Brazil. The analyzed variables were dimensions of the fibers (length, total diameter and diameter of the lumen and wall thickness), the vessel elements (tangential diameter and frequency), and ray (height, width and frequency). The specific gravity (basic, at $12 \%$ moisture content and dry), and total volumetric and linear shrinkages, resistance to bending, compression parallel to the fibers, shear and Janka hardness. For anatomical description it was found numerous vessels often with small tangential diameter, heterocellular rays occurring at low frequency, and long libriform fibers with thick to thin walls. The specific gravity of wood from disks taken at $1.30 \mathrm{~m}$ from ground level was $0.48 \mathrm{~g} \mathrm{~cm}^{-3}$, wich we considered average, the dimensional stability was low, and there was great influence of moisture in the resistance values in wood.

Keywords: Anatomy; specific gravity; dimensional stability; mechanical strength.
\end{abstract}

\section{INTRODUÇÃO}

O setor florestal brasileiro vem se destacando no cenário mundial por causa da diversidade das florestas nativas e da capacidade produtiva das florestas plantadas, juntamente com a demanda por madeira. $\mathrm{O}$ alto consumo de madeiras oriundas de florestas nativas, durante muito tempo, levou a quase extinção de muitas espécies de alto valor econômico. Uma alternativa para o suprimento dessa demanda tem sido a substituição de madeiras nativas por aquelas provenientes de reflorestamento, pois além de contribuir para a diminuição do desmatamento, possuem um desenvolvimento rápido e características adequadas para novos usos (SOUSA, 2004), como produtos sólidos de madeira e painéis reconstituídos.

FLORESTA, Curitiba, PR, v. 45, n. 4, p. 723 - 734, out. / dez. 2015.

Freitas, T. P. et al.

ISSN eletrônico 1982-4688 / ISSN impresso 0015-3826 
O gênero Liquidambar, da família Altingiaceae, popularmente conhecido como liquidâmbar ou "sweetgum"; é constituído por quatro espécies intercontinentais na zona temperada do Hemisfério Norte. Duas espécies são encontradas na Ásia oriental (L. formosana e L. acalycina), uma na Ásia ocidental ( $L$. orientalis) e a outra entre a América do Norte oriental e central (L. styraciflua) (PIGG et al., 2003; ICKERT-BOND et al., 2005).

Suas árvores, com folhas caducifólias, apresentam rápido crescimento, são altamente resistente ao ataque de insetos, possuem tronco retilíneo, e podem atingir uma altura de $45 \mathrm{~m}$ e diâmetros de $1,2 \mathrm{~m}$ na idade adulta (SEBBENN et al., 2007; KORMANIK 1990).

Vários autores (MCCARTUR, HUGHES, 1984; KORMANIK, 1990; SHIMIZU, 2005) constataram a boa qualidade da madeira de Liquidambar, descrevendo seus múltiplos usos, como matéria prima para celulose, dormentes, madeira serrada para embalagens, estrados, móveis, lenha, e especialmente em indústrias de laminados e compensados. Segundo Shimizu (2005), outras utilidades destas espécies são para paisagismo e arborização urbana, devido à beleza estética de suas árvores com uma intensa coloração das folhas no outono.

Acredita-se que espécies do gênero Liquidambar podem ser utilizadas como alternativa para as indústrias brasileiras, principalmente como madeira serrada, para substituir espécies utilizadas atualmente, como eucalipto e pinus (LIMA et al., 2013). Plantios experimentais destas espécies localizados nas regiões Sul e Sudeste do Brasil comprovaram sua capacidade de adaptação e crescimento, e sua utilidade para usos múltiplos (SHIMIZU, 2005). Shimizu e Spir (2004) ao estudarem a produtividade da madeira de Liquidambar styraciflua L. aos 11 anos de idade, em Quedas do Iguaçu, encontraram uma produtividade média de até $40 \mathrm{~m}^{3} \mathrm{ha}^{-1}$ ano $^{-1}$.

O desenvolvimento das árvores é influenciado pelas características genotípicas, pelas qualidades do sítio e influências climáticas. Consequentemente, a madeira como produto oriundo do seu crescimento irá apresentar estrutura variável, com diferentes propriedades anatômicas e físico-mecânicas. Sendo assim, é importante o conhecimento de tais propriedades para um melhor aproveitamento do material.

Portanto, tratando-se de uma árvore exótica, com boa capacidade de adaptação e crescimento em regiões de clima ameno no Brasil, porém pouco estudada, torna-se importante o conhecimento das características tecnológicas de sua madeira, com a finalidade de se obter informações sobre suas possíveis utilizações. Assim, este trabalho teve como objetivo a caracterização anatômica e físico-mecânica da madeira Liquidambar sp.

\section{MATERIAL E MÉTODOS}

\section{Coleta e caracterização anatômica do material}

O material utilizado foi proveniente de três árvores de Liquidambar sp.com idade de oito anos, tendo altura total de $15,7 \mathrm{~m}$ e diâmetro à altura do peito (DAP), de $23,3 \mathrm{~cm}$, provenientes de um arboreto, em espaçamento de 3 × 3 m, localizado no município de Guaçuí, Espírito Santo, nas coordenadas - $41^{\circ}$ 40 " $46^{\prime}$ " de longitude e $-20^{\circ} 46^{\prime} 32$ " de latitude, a uma altitude de 590 metros.

Para a caracterização geral e macroscópica da madeira utilizou-se o manual de descrição das características gerais e macroscópicas de madeiras de angiospermas dicotiledôneas da Comisión Panamericanca de Normas Técnicas (COPANT, 1974). A cor da madeira seca ao ar foi determinada de acordo com a escala de Munsell (1957), e para o estudo microscópico seguiram-se as recomendações da norma de procedimento em estudos de anatomia da madeira, da COPANT (1974) e também a lista de características microscópicas para a identificação de madeira da International Association of Wood Anatomists (IAWA, 1989).

Uma amostra de cada árvore foi retirada na zona de transição entre cerne e alburno, na região correspondente ao DAP. Para a mensuração dos vasos e raios, foram utilizados três corpos de prova com dimensões de $1,0 \times 1,5 \times 2,0 \mathrm{~cm}$, retirados nos planos longitudinais (radial e tangencial) e transversal em relação ao crescimento do tronco.

As amostras obtidas foram amolecidas em água à temperatura de ebulição, e fixadas em micrótomo de deslize, para obtenção de cortes histológicos com espessura variando de 18 a $20 \mu \mathrm{m}$. Os cortes foram montados em lâminas temporárias, com a utilização de glicerina e água destilada na proporção de 1:1. 
Foram obtidas fotomicrografias dos cortes, pelo emprego de uma câmera fotográfica digital acoplada a um microscópio óptico. Com auxílio de um sistema de análises de imagem provido do software Image Pro Express 6.0, foi possível mensurar a frequência vascular (número $\mathrm{mm}^{-2}$ ), diâmetro tangencial dos poros $(\mu \mathrm{m})$ e para os raios foram mensurados a altura, largura ( $\mu \mathrm{m}$ e número de células) e frequência (número $\mathrm{mm}^{-1}$ ).

Para a dissociação dos elementos anatômicos para mensuração das fibras, foi utilizado o método proposto por Nicholls e Dadswell descrito por Ramalho (1987). Para tanto, foram obtidos do plano radial das amostras fragmentos de madeira que foram transferidos para frascos tipo penicilina, com capacidade de $25 \mathrm{ml}$ contendo solução de ácido acético e peróxido de hidrogênio na proporção 1:1.

Os frascos após serem lacrados, foram transferidos para estufa, mantidos a uma temperatura de $60{ }^{\circ} \mathrm{C}$, durante 48 horas. Depois de realizada a lavagem da solução macerante em água corrente, os cortes foram corados com solução alcoólica de $1 \%$ de safranina e as lâminas histológicas montadas, com uso de água destilada e glicerina na proporção de 1:1, e obtidas as fotomicrografias como mencionado anteriormente. Então, foram mensurados o comprimento, a largura da fibra $(\mu \mathrm{m})$ e o diâmetro do lume $(\mu \mathrm{m})$ e a espessura da parede celular $(\mu \mathrm{m})$ foi obtida pela diferença entre a largura da fibra e o diâmetro do lume, dividida por dois.

\title{
Massa específica e estabilidade dimensional da madeira
}

Para a determinação da massa específica básica da madeira no sentido longitudinal da árvore retirou-se um disco de $4 \mathrm{~cm}$ de espessura para cada posição a $0,25,50,75$ e $100 \%$ da altura comercial da árvore, e um disco no DAP (Figura 1). De cada disco, de cada posição, foram retiradas e analisadas duas cunhas localizadas em sentidos opostos, a fim de verificar a variação da massa específica básica no sentido longitudinal. Para a variação no sentido radial (medula-casca) retirou-se um disco suplementar no DAP (Figura 1), do qual foi obtida uma bagueta no sentido radial, que foi transformada em pequenos corpos de prova retirados em intervalos de $1,0 \mathrm{~cm}$ a partir da medula, em direção à casca.

Para as determinações da massa específica aparente e anidra da madeira foram utilizados 20 corpos de prova com dimensões $2,0 \times 2,0 \times 3,0 \mathrm{~cm}$ (radial x tangencial x longitudinal), retirados de toretes de um metro obtidos da região abaixo e acima do DAP (Figura 1). Para obtenção dos corpos de prova, dos toretes obtidos, foram retiradas duas costaneiras, obtendo-se um pranchão central de 10 a 12 $\mathrm{cm}$ de espessura (Figura 1).

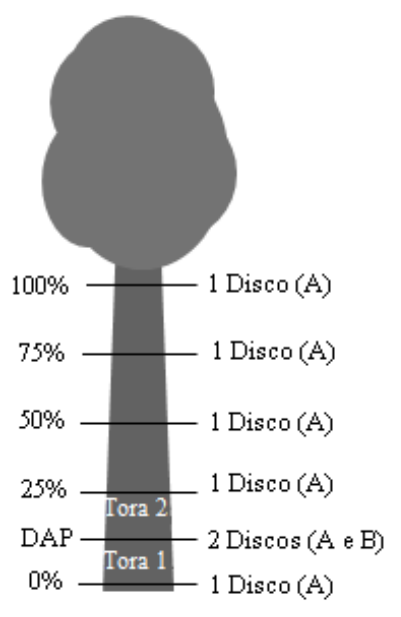

\author{
Disco A - Massa especifica básica no \\ sentido longitudinal
}

Disco B - Massa específica básica no sentido radial e característica anatômica

Toras 1 e 2 - Massa especifica aparente, estabilidade dimensional e propriedades mecânicas

Figura 1. Esquema de amostragem dos discos e toras nas árvores.

Figure 1. Sampling design of disks and $\operatorname{logs}$ in the trees.

As massas específicas básica, aparente e anidra foram determinadas de acordo com as Normas Brasileiras Regulamentadoras NBR 6230 e NBR 11941 da Associação Brasileira de Normas Técnicas (ABNT) $(1985 ; 2003)$.

Para determinar as contrações lineares radial, tangencial, longitudinal e volumétrica foram empregados 20 corpos de prova com dimensões de 2,0 x 2,0 x 3,0 cm (radial x tangencial x longitudinal),

FLORESTA, Curitiba, PR, v. 45, n. 4, p. 723 - 734, out. / dez. 2015.

Freitas, T. P. et al.

ISSN eletrônico 1982-4688 / ISSN impresso 0015-3826 
retirados dos mesmos toretes. As determinações foram realizadas de acordo com a NBR 6230 (ABNT, 1985). As medições das dimensões dos corpos de prova foram obtidas com a madeira verde (acima de $28 \%$ de umidade) e secas, após colocadas em estufa, inicialmente a $60{ }^{\circ} \mathrm{C}$, incrementando gradativamente a temperatura de $20^{\circ} \mathrm{C}$ a cada 24 horas até $103 \pm 2^{\circ} \mathrm{C}$, permanecendo nestas condições até adquirirem massas constantes.

\section{Propriedades mecânicas da madeira}

Os corpos de prova para os ensaios mecânicos foram confeccionados do pranchão central obtido. Os ensaios foram realizados nos teores de umidade verde e seca ao ar, sendo utilizados metade da quantidade dos corpos de prova em estado verde e os demais colocados para secar à sombra, em local coberto, até atingir a umidade de equilíbrio.

Para cada condição de umidade, as repetições e dimensões (largura $\mathrm{x}$ espessura $\mathrm{x}$ comprimento) dos corpos de provas foram de $40(2 \times 2 \times 30 \mathrm{~cm}) ; 40(2 \times 2 \times 3 \mathrm{~cm}) ; 24(5 \times 5 \times 6,4 \mathrm{~cm})$ e $6(6 \times 6 \times$ $15 \mathrm{~cm}$ ) para os ensaios de flexão estática (Módulo de Ruptura (MOR) e Módulo de Elasticidade (MOE)), resistência à compressão paralela às fibras, ao cisalhamento e dureza Janka, respectivamente, de acordo com a NBR 6230 (ABNT, 1985).

Depois de realizados os ensaios mecânicos, algumas amostras de cada ensaio foram retiradas para determinação do seu teor de umidade, objetivando o ajuste dos resultados ao padrão de $12 \%$, conforme especificação da NBR 7190 da ABNT (1997).

\section{RESULTADOS E DISCUSSÃO}

\section{Características organolépticas, macroscópicas e microscópicas da madeira}

A madeira possui cerne e alburno indistintos, coloração marrom pálida 10YR 7/3 de acordo com a escala de Munsell (1957). Não possui brilho nem odor, sendo macia ao corte manual no plano transversal, possui grã direita e textura fina e suas camadas de anéis de crescimento são distintas (Figura 2). A madeira de Liquidambar sp. tem parênquima axial invisível mesmo sob lente de aumento (10 vezes). Os poros e os raios são imperceptíveis a olho nu.

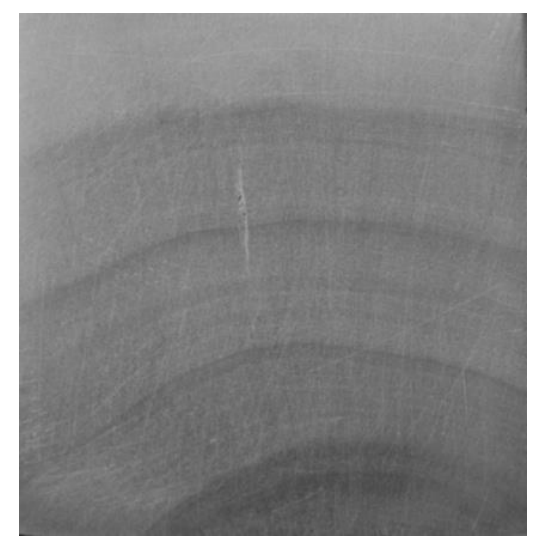

Figura 2. Ilustração das camadas de anéis de crescimento da madeira de Liquidambar sp.

Figure 2. Illustration of the layers of wood growth rings of Liquidambar sp.

Os poros possuem diâmetro tangencial médio de $44,80 \mu \mathrm{m}$, sendo classificados como pequenos (Tabela 1), com distribuição difusa, são solitários, de seção ovalada a circular, e não obstruídos. Segundo Chimelo (2007) madeiras que possuem vasos ou poros de pequeno diâmetro dão bom acabamento com vernizes e tintas. Quanto à frequência vascular são muito numerosos, com média de 66,16 poros por $\mathrm{mm}^{2}$, variando de 53 a 83 poros por $\mathrm{mm}^{2}$ e coeficiente de variação de $11,47 \%$ (Tabela 1). As placas de perfuração são múltiplas do tipo escalariformes (Figura 3), pontoações intervasculares alternas com presença de apêndices em ambas as extremidades e possui parênquima axial apotraqueal difuso e paratraqueal escasso. 

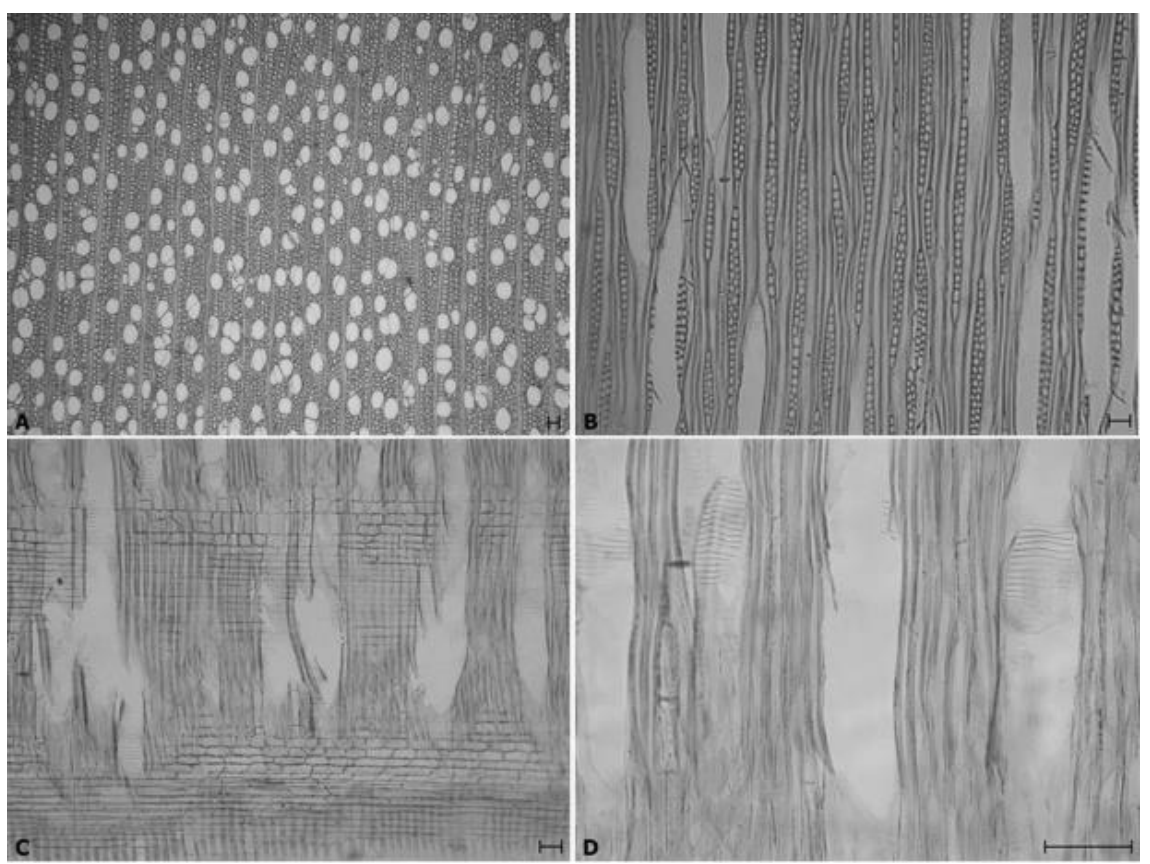

Figura 3. Secão transversal (A), tangencial (B) e radial (C) da madeira de Liquidambar sp. Placas de perfuração múltiplas escalariformes na seção radial (D). Escala: $100 \mu \mathrm{m}$.

Figure 3. Transverse (A) tangential (B) and radial (C) sections of the Liquidambar sp. wood. Multiperforation plate scalariform in the radial section (D). Scale bar $=100 \mu \mathrm{m}$.

Tabela 1. Mensuração de elementos anatômicos do cerne periférico, tomado no diâmetro a altura do peito (DAP) da madeira de Liquidambar sp.

Table 1. Measurement of anatomical elements of the Liquidambar sp.peripheral heartwood, taken in the diameter at breast height (DBH).

\begin{tabular}{llcccc}
\hline \multicolumn{1}{l}{ Caracteres anatômicos } & Mínimo & Máximo & Média & CV(\%) \\
\hline Vasos & Diâmetro Tangencial $(\mu \mathrm{m})$ & 25,26 & 51,41 & 44,80 & 30,30 \\
& Frequência $\left(\right.$ vasos $\left.\mathrm{mm}^{-2}\right)$ & 53 & 83 & 66,16 & 11,47 \\
\hline \multirow{2}{*}{ Raios } & Altura $(\mu \mathrm{m})$ & 138,98 & 850,32 & 404,09 & 35,99 \\
& Altura (número células) & 6 & 43 & 18,61 & 35,60 \\
& Largura $(\mu \mathrm{m})$ & 12,63 & 41,17 & 25,07 & 27,42 \\
& Largura (número células) & 1 & 3 & 1,91 & 24,65 \\
& Frequência (número mm $\left.{ }^{-1}\right)$ & 6 & 12 & 8,73 & 15,79 \\
\hline Fibras & Comprimento $(\mu \mathrm{m})$ & 1300,72 & 2640,42 & 1980,88 & 14,74 \\
& Largura $(\mu \mathrm{m})$ & 21,78 & 52,84 & 30,83 & 15,64 \\
& Diâmetro Lume $(\mu \mathrm{m})$ & 9,45 & 38,04 & 15,70 & 26,46 \\
& Espessura Parede $(\mu \mathrm{m})$ & 5,63 & 10,54 & 7,56 & 13,01 \\
\hline
\end{tabular}

$\mathrm{CV}=$ Coeficiente de variação.

Os raios são heterocelulares, possuem estrutura não estratificada e são formados por células procumbentes e eretas, sendo as eretas situadas na extremidade superior. $\mathrm{O}$ parênquima radial possui frequência média de 8,73 raios por $\mathrm{mm}$, variando de 6 a 12 raios por $\mathrm{mm}$, com coeficiente de variação de 15,79\%. São baixos, com altura média de $404,09 \mu \mathrm{m}$, altura mínima de $138,98 \mu \mathrm{m}$ e máxima de $850,32 \mu \mathrm{m}$. A altura média em número de células foi de 18,61 células, variando de 6 a 43 células.

Quanto à largura dos raios, o valor médio foi de 25,07 $\mu \mathrm{m}$, com largura mínima de 12,63 $\mu \mathrm{m}$ e máxima de 41,17 $\mu \mathrm{m}$, caracterizando-os como finos. Já para a largura dos raios em número de células a variação foi de 1 a 3 células, com valor médio de 1,91 células de largura, sendo classificados como multisseriados. 
A madeira possui fibras libriformes, com comprimento médio de 1980,88 $\mu$ m no cerne periférico, sendo classificadas como longas, variando de curtas $(1300,72 \mu \mathrm{m})$ a longas $(2640,42 \mu \mathrm{m})$. Os valores encontrados para o comprimento das fibras estão próximos dos registrados por Mattos et al. (2001) que ao estudarem as características da madeira de Liquidambar styraciflua proveniente de um arboreto localizado em Colombo, Paraná, aos dezesseis anos de idade, encontraram valores médios de $1550 \mu \mathrm{m}$.

Quanto à largura das fibras, o valor médio foi de $30,83 \mu \mathrm{m}$. O diâmetro médio do lume de $15,70 \mu \mathrm{m}$. A espessura da parede teve valor médio de 7,56 $\mu \mathrm{m}$, sendo, portanto, fibras de paredes delgadas a espessas (IAWA, 1989).

O estudo anatômico da madeira tem como principal objetivo verificar a relação entre as características estruturais da madeira e seu uso. As dimensões das fibras, por exemplo, são indicadores importantes das propriedades de uma determinada espécie de madeira e sua adequação para a fabricação de papel (HUGHES, 1973; SILVA, 2011). Os principais parâmetros considerados são o comprimento, a relação comprimento: largura e a espessura da parede da fibra (FOELKEL, 2007; MENEGAZZO, 2012).

O comprimento das fibras está relacionado com a resistência das fibras celulósicas, e afeta algumas propriedades, como resistência ao rasgo e resistência a dobras. Fibras mais longas na celulose possuem maior coarseness, o que significa menor número de fibras por unidade de massa, conferindo maior resistência à celulose quando comparada com fibras curtas (MENEGAZZO, 2012). A espessura da parede esta relacionada com a rigidez da fibra, sendo que fibras com maiores espessuras de parede podem sofrer maior desfibrilamento durante o processo de refinação, aumentando o potencial de ligações interfibrilares, sua área de ligação e, consequentemente a resistência à tração do papel (CARVALHO et al., 1998; SANTOS, 2005).

Desta forma, com base nas propriedades anatômicas da madeira de Liquidambar sp.,acredita-se que a mesma possa ser utilizada para produção de celulose, por possuir fibras de comprimento longo e de paredes delgadas a espessa, porém, é necessária uma avaliação das propriedades químicas desta madeira para fundamentar esta utilização.

\section{Massa específica e estabilidade dimensional da madeira}

Os baixos valores de coeficiente de variação para as posições analisadas ao longo do tronco indicam uma baixa variabilidade da massa específica no sentido longitudinal para as árvores avaliadas, que não obtiveram grande amplitude de variação em torno do valor médio de $0,49 \mathrm{~g} \mathrm{~cm}^{-3}$, com variação máxima de 0,05 obtida entre as posições de 25 e $100 \%$ da altura comercial (Figura 4). Este resultado também foi observado por Mattos et al. (2001).

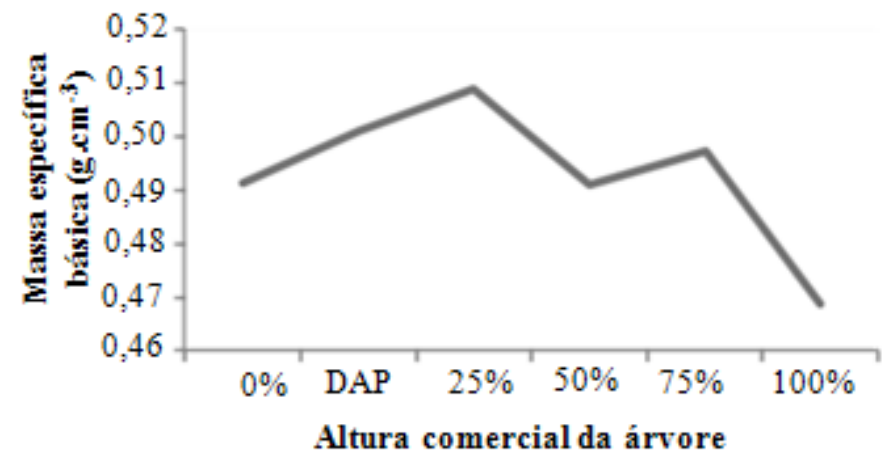

Figura 4. Variação longitudinal da massa específica básica da madeira de Liquidambar sp. Figure 4. Longitudinal variation of the basic specific gravity of the Liquidambar sp.wood.

Pela observação da figura 4, nota-se que a massa específica tende a aumentar até próximo a $25 \%$ da altura comercial da árvore, alcançando os maiores valores neste ponto e a partir dele tende a diminuir até $100 \%$ da altura comercial.

Os dados evidenciam uma pequena variação na massa específica básica na direção radial, com variação máxima de 0,06 obtida entre as posições $3 ; 5$ e 1;8 o que leva a inferir uma homogeneidade da 
madeira. Tal comportamento pode ser explicado pela idade precoce das árvores, o que poderá ser caracterizado pela presença única de formação de madeira juvenil.

Segundo Oliveira et al. (2005), madeiras mais homogêneas, quanto à sua massa específica no interior do tronco, podem se comportar melhor nas operações de processamento e refletir em maior uniformidade nas demais propriedades tecnológicas.

Quanto à variação da massa específica básica na direção radial do tronco, observa-se que a mesma aumentou no sentido medula-casca da árvore até a posição 3 e depois diminui nas posições mais próximas da casca (Figura 5).

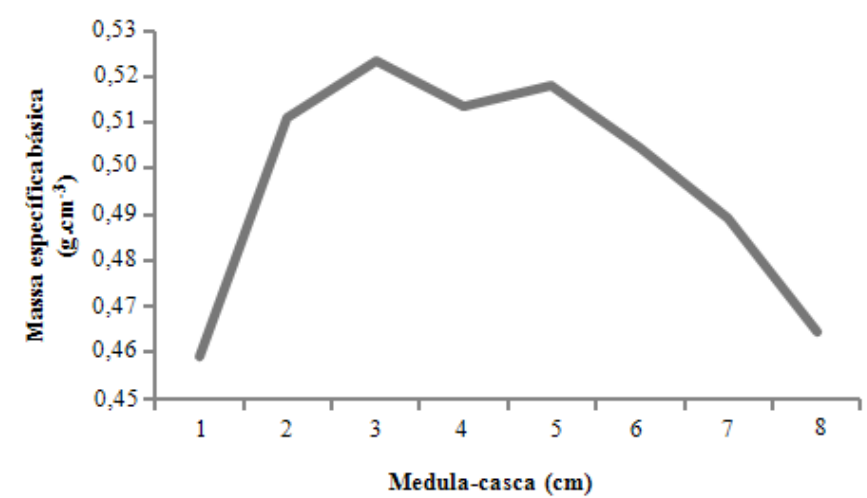

Figura 5. Variação radial da massa específica básica da madeira de Liquidambar sp.

Figure 5. Radial variation of the basic specific gravity of the Liquidambar sp. wood.

De acordo com a tabela 2, o valor médio de massa específica básica da madeira para as árvores de Liquidambar sp. na região do DAP foi de $0,48 \mathrm{gcm}^{-3}$, que a classifica como de média massa específica de acordo com a COPANT (1974).

Tabela 2. Propriedades físicas da madeira de Liquidambar sp.

Table 2. Physical properties of the Liquidambar sp. wood.

\begin{tabular}{|c|c|c|c|c|}
\hline \multicolumn{2}{|c|}{ Propriedades Físicas } & Média & Mínimo & Máximo \\
\hline \multirow{3}{*}{$\begin{array}{l}\text { Massa Específica } \\
\text { Aparente } \\
\left(\mathrm{g} \mathrm{cm}^{-3}\right)\end{array}$} & Básica & $\begin{array}{c}0,48 \\
(2,89)\end{array}$ & 0,46 & 0,51 \\
\hline & $12 \%$ de umidade & $\begin{array}{c}0,55 \\
(3,22)\end{array}$ & 0,52 & 0,59 \\
\hline & Anidra & $\begin{array}{c}0,59 \\
(3,38) \\
(5,94) \\
\end{array}$ & 0,55 & 0,63 \\
\hline \multirow[t]{4}{*}{$\begin{array}{l}\text { Contração Total } \\
(\%)\end{array}$} & Radial & $\begin{array}{c}5,53 \\
(8,67)\end{array}$ & 4,41 & 6,28 \\
\hline & Tangencial & $\begin{array}{l}15,24 \\
(5,26)\end{array}$ & 14,05 & 17,26 \\
\hline & Longitudinal & $\begin{array}{c}1,28 \\
(53,10)\end{array}$ & 0,38 & 2,63 \\
\hline & Volumétrico & $\begin{array}{l}21,79 \\
(4,67)\end{array}$ & 20,07 & 24,41 \\
\hline \multicolumn{2}{|c|}{ Fator Anisotrópico } & $\begin{array}{c}2,77 \\
(10,09)\end{array}$ & 2,33 & 3,49 \\
\hline
\end{tabular}

Valores entre parênteses correspondem ao coeficiente de variação (\%).

O Forest Products Laboratory (FPL) (2010) cita, para a madeira de Liquidambar sp., um valor de massa específica básica semelhante ao obtido nesta pesquisa $\left(0,46 \mathrm{~g} \mathrm{~cm}^{-3}\right)$. Massa específica básica de

FLORESTA, Curitiba, PR, v. 45, n. 4, p. 723 - 734 , out. / dez. 2015.

Freitas, T. P. et al.

ISSN eletrônico 1982-4688 / ISSN impresso 0015-3826

DOI: $10.5380 /$ rf.v45i4.36878 
$0,52 \mathrm{~g} \mathrm{~cm}^{-3}$ foi encontrada por Mattos et al. (2001), para a madeira de Liquidambar styraciflua aos 16 anos de idade.

No entanto, Carpenter e Hopkins (1966), ao estudarem o comportamento da densidade básica na parte superior do tronco comparado à inferior para a madeira de Liquidambar styraciflua, com 50 anos de idade em Mississipi, Estados Unidos, encontraram valor médio de densidade básica de $0,51 \mathrm{~g} \mathrm{~cm}^{-3}$.

Jeffries (2008), ao estudar as correlações da taxa de crescimento e das propriedades mecânicas de Liquidambar styraciflua, em dois locais nos Estados Unidos, Pensacola (Florida) e Summerville (Carolina do Sul), com 9 anos de idade, encontrou para esta madeira, uma densidade básica média de $0,56 \mathrm{~g} \mathrm{~cm}^{-3}$. As diferenças encontradas podem ser explicadas pela idade das árvores e pela qualidade do sítio. Geralmente a massa específica da madeira, tende a aumentar com a idade.

Para a massa específica aparente da madeira de Liquidambar sp. na umidade de $12 \%$, o valor médio encontrado foi de $0,55 \mathrm{~g} \mathrm{~cm}^{-3}$. Este valor foi próximo ao citado pelo FPL (2010), que obteve para esta madeira massa específica aparente de $0,52 \mathrm{~g} \mathrm{~cm}^{-3}$. O valor médio de massa específica anidra para a madeira na região do DAP foi $0,59 \mathrm{~g} \mathrm{~cm}^{-3}$. As diferenças podem estar relacionadas com as características edafoclimáticas das regiões de procedência das madeiras.

Segundo Chimelo (2007), as fibras são os elementos celulares que mais se relacionam com a massa específica do lenho e, madeiras com fibras de paredes espessas geralmente possuem alta massa específica. A madeira estudada possui massa específica básica média, e fibras com parede delgadas a espessas, Assim os valores de massa específica foram influenciados, além da espessura de parede das fibras, pela abundância (frequência) vascular e pela ocorrência de vasos de pequenos diâmetros.

Quanto à estabilidade dimensional, a madeira em estudo obteve valores médios para contração radial, tangencial, longitudinal e volumétrico de 5,53, 15,24, 1,28 e 21,79\% respectivamente. Segundo Oliveira (2007), a variação dimensional da madeira varia conforme a espécie, mas em média os valores de contração radial, tangencial e longitudinal, variam de 2-9; 4-20 e 0,1-0,3\%, respectivamente, com a contração volumétrica variando de $8-26 \%$. Portanto, verifica-se que as contrações volumétrica, radial e tangencial, estão dentro do intervalo apresentado, com exceção da contração longitudinal com valor médio de $1,28 \%$.

Mattos et al. (2001), encontraram para esta madeira valores próximos de contração radial e tangencial, de 5,4 e 12,7\%, respectivamente, e valores superiores para contração longitudinal e volumétrica, de 0,4 e 18,5\%, respectivamente. De acordo com o FPL (2010), para a madeira de Liquidambar sp. as contrações radial, tangencial e volumétrica são de 5,3; 10,2 e 15,8\%. O fator anisotrópico encontrado foi igual a 2,77. Mattos et al. (2001), encontraram em seu estudo fator anisotrópico de 2,3, para a madeira de Liquidambar styraciflua aos 16 anos de idade.

Segundo Oliveira (2007), o coeficiente anisotrópico varia de 1,3 a 1,4 para madeiras muito estáveis, a mais de 3, para espécies extremamente instáveis dimensionalmente. Logo, a madeira deste estudo é considerada de baixa estabilidade dimensional, portanto, de um modo geral, poderá ser utilizada em aplicações onde esta característica não seja importante.

\section{Propriedades mecânicas da madeira}

Ao comparar valores dos módulos de ruptura (MOR) e de elasticidade (MOE) obtidos para a madeira no estado verde com aqueles encontrados para a madeira a $12 \%$ de umidade, observa-se um aumento dos valores, o que evidencia a influência da umidade nestas propriedades (Tabela 3).

Segundo Oliveira (2007), a secagem melhora as propriedades mecânicas da madeira, podendo uma madeira com $12 \%$ de umidade possuir o dobro de sua resistência quando no estado verde. Portanto os acréscimos no MOR e MOE da madeira de Liquidambar sp.causados pela secagem são normais e desejáveis.

Para a madeira de Liquidambar sp. com massa específica básica de $0,46 \mathrm{~g} \mathrm{~cm}^{-3}$, o FPL (2010) encontrou valor médio para MOR igual a 49 e $86 \mathrm{MPa}$, e para MOE valor médio igual a 8.300 e 11.300 MPa, na umidade verde e a $12 \%$, respectivamente.

Para o presente estudo houve grande influência da umidade na resistência, e algumas propriedades como MOR e dureza Janka paralela às fibras demonstraram um incremento maior que o dobro da resistência para testes realizados a $12 \%$, quando comparados a madeira testada no estado verde, com aumentos de 119,71 para o MOR e de $126,46 \%$ para a dureza. 
Tabela 3. Propriedades mecânicas da madeira de Liquidambar sp. de acordo com a umidade.

Table 3. Mechanical properties of theLiquidambar sp. wood according to moisture content.

\begin{tabular}{|c|c|c|c|}
\hline \multirow{2}{*}{ Propriedades mecânicas } & & \multicolumn{2}{|c|}{ Umidade } \\
\hline & & Verde & $12 \%$ \\
\hline \multirow[t]{4}{*}{ Flexão Estática } & & 46,20 & 101,60 \\
\hline & MOR (MPa) & $(9,17)$ & $(9,68)$ \\
\hline & MOE (MPa) & $\begin{array}{l}5.282,0 \\
(11,74)\end{array}$ & $\begin{array}{l}7.652,7 \\
(12,86)\end{array}$ \\
\hline & Trabalho (J) & $\begin{array}{c}34 \\
(26,24)\end{array}$ & $\begin{array}{c}18 \\
(34,53)\end{array}$ \\
\hline Compressão axial & Limite de resistência (MPa) & $\begin{array}{c}28,9 \\
(13,79)\end{array}$ & $\begin{array}{c}56,7 \\
(13,60)\end{array}$ \\
\hline Cisalhamento & Limite de resistência (MPa) & $\begin{array}{c}8,6 \\
(14,15)\end{array}$ & - \\
\hline \multirow[t]{3}{*}{ DurezaJanka } & $\begin{array}{c}\text { Paralela às fibras } \\
(\mathrm{MPa})\end{array}$ & $\begin{array}{c}31,6 \\
(5,56)\end{array}$ & $\begin{array}{c}71,6 \\
(4,17)\end{array}$ \\
\hline & $\begin{array}{l}\text { Radial } \\
(\mathrm{MPa})\end{array}$ & $\begin{array}{c}36,3 \\
(4,85)\end{array}$ & $\begin{array}{c}46,4 \\
(7,13)\end{array}$ \\
\hline & $\begin{array}{l}\text { Tangencial } \\
(\mathrm{MPa})\end{array}$ & $\begin{array}{c}33,3 \\
(2,94)\end{array}$ & $\begin{array}{c}57,5 \\
(16,40)\end{array}$ \\
\hline
\end{tabular}

Valores entre parênteses correspondem ao coeficiente de variação (\%).

Para a resistência à compressão paralela às fibras, obteve-se, valor médio na umidade verde e a $12 \%$ igual a 28,90 e $56,70 \mathrm{MPa}$, respectivamente, o que correspondeu a um acréscimo de $96 \%$. FPL (2010) encontrou valores de 21,00 e 43,60 MPa, para a madeira de Liquidambar sp. em estado verde e a $12 \%$ de umidade, respectivamente.

Com relação à resistência ao cisalhamento, notou-se que o valor médio obtido para a madeira na umidade verde foi igual a 8,64 MPa. Valores inferiores foram encontrados pelo FPL (2010), que obteve para a resistência ao cisalhamento na umidade verde um valor médio de 6,80 MPa.

Os valores médios para dureza Janka, na direção paralela às fibras e nas direções radial e tangencial, na umidade verde e a $12 \%$ foram de 31,60 e 71,60 MPa, 36,30 e 46,40 MPa, 33,33 e $57,51 \mathrm{MPa}$, respectivamente. Estes resultados foram superiores aos obtidos pelo FPL (2010), para esta madeira em estado verde e a $12 \%$ de umidade, que obteve dureza Janka de 27,0 e 38,0 MPa, respectivamente.

\section{CONCLUSÕES}

- Para a madeira de Liquidambar sp. com 8 anos, constatou-se numerosa frequência vascular de pequeno diâmetro tangencial, raios heterocelulares com pouca frequência, e fibras libriformes longas de paredes delgadas a espessas.

- As árvores possuem pequena variação quanto à massa específica básica da madeira, tanto no sentido longitudinal quanto radial.

- A madeira possui massa específica aparente média, estabilidade dimensional baixa, com altos valores de contração volumétrica e de coeficiente de anisotropia.

- Os valores de resistência mecânica da madeira de Liquidamba sp. foram influenciados pela umidade. A resistência da madeira a $12 \%$ de umidade, para as características avaliadas, foi praticamente o dobro da resistência da madeira no estado verde.

- No estágio de desenvolvimento das plantas avaliadas, sua madeira, em decorrência da baixa estabilidade dimensional, não é indicada para a produção de móveis, apesar de possuir características desejáveis para este uso, como grã direita, textura fina e ausência de odores.

\section{REFERÊNCIAS}

ASSOCIAÇÃO BRASILEIRA DE NORMAS TÉCNICAS (ABNT) NBR 7190: Projeto de estruturas de madeira. Projeto de Revisão NBR7190. Rio de Janeiro, 1997. 60p.

FLORESTA, Curitiba, PR, v. 45, n. 4, p. 723 - 734 , out. / dez. 2015.

Freitas, T. P. et al.

ISSN eletrônico 1982-4688 / ISSN impresso 0015-3826

DOI: $10.5380 /$ rf.v45i4.36878 
NBR 6230: métodos de ensaio para madeiras. Rio de Janeiro, 1985. 89 p.

NBR 11941: madeira - determinação da densidade básica. Rio de Janeiro, 2003. 6 p.

CARVALHO, H. G.; OLIVEIRA, R. C.; GOMIDE, J. L.; COLODETTE, J. L. Efeito da idade de corte da madeira e de variáveis de refino nas propriedades da celulose kraft branqueada de eucalipto. In: CONGRESSO ANUAL DE CELULOSE E PAPEL DA ABTCP, 31., São Paulo, 1998. Anais... São Paulo: ABTCP, 1998, p. 367 - 381.

CARPENTER, B. E.; HOPKINS, W. C. Specific gravity values of sweetgum (Liquidambar styraciflua) topwood and bolewood.Forest Products Journal, Madison, v. 6, n. 7, p. 30, 1966.

CHIMELO, J. Anatomia e propriedades gerais da madeira. In: OLIVEIRA, J. T. S.; FIEDLER, N. C.; NOGUEIRA, M. (Eds.). Tecnologias aplicadas ao setor madeireiro. Visconde do Rio Branco: Suprema Gráfica e Editora, 2007.p. 107 - 124.

COMISIÓN PANAMERICANCA DE NORMAS TÉCNICAS (COPANT). Descripción de características generales, macroscópicas de las maderas angiospermas dicotiledoneas. Buenos Aires: COPANT, v. 30, p. 1 - 19, 1974.

FOELKEL, C. As fibras dos eucaliptus e as qualidades requeridas na celulose Kraft para fabricação de papel. In: Eucalyptus Online Book, 2007. 48 p.

FOREST PRODUCTS LABORATORY (LPF). Wood handbook: wood as an engineering material. $100^{\text {th }}$ ed. Madison: U.S. Department of Agriculture, Forest Service, Forest Products Laboratory, 2010. 508 p. (General Technical Report FPL-GTR, 190).

HUGHES, J. F. The wood structure of Pinus caribea Morelet in relation to use characteristics, growth conditions and tree improvement. In: BURLEY, J.; NIKKLES, D. G. (Ed.). Selection and breeding to improve some tropical conifers. Oxford: Commonwealth Forestry Institute, 1973. p. 13 - 22.

INTERNATIONAL ASSOCIATION OF WOOD ANATOMISTS (IAWA COMMITTEE). List of microscopic features for hardwood identification, with an appendix on non-anatomical information. IAWA Bulletin, Leiden, v. 10, n. 3, p. 219 - 332, 2007.

ICKERT-BOND, S. M.; PIGG, K. B.; WEN, J. Comparative infructescence morphology in Liquidambar (Altingiaceae) and its evolutionary significance. American Journal of Botany, St. Louis, v. 92, p. 1234 1255,2005 .

JEFFRIES, T. M. Relationships of growth rate and mechanical properties in sweetgum, Liquidambar styraciflua. 2008, 98 f. Dissertation (Master Science in Forest Products) - Faculty of the Virginia Polytechnic Institute and State University, Blacksburg, 2008.

KORMANIK, P. P. Liquidambar styraciflua L. Sweetgum.In: Burns RM, Honkala BH (Eds.). Silvics of North America: Hardwoods. Washington; 1990, p. 400 - 405.

LIMA, I. L.; FREITAS, J. Á.; GARCIA, J. N. Influência da classe diamétrica nos índices de rachadura da madeira em Liquidambar styraciflua. Floresta e Ambiente, Seropédica, v. 20, n. 1, p. 117 - 123, 2013.

MATTOS, P. P.; PEREIRA, J. C. D.; SCHAITZA, E. G.; CARVALHO, P. E. R. Características da madeira de Liquidambar styraciflua. Circular Técnica Embrapa Florestas, Colombo, v. 49, p. 1 - 4, 2001.

MCCARTER, O. S.; HUGHES, C. E. Liquidambar styraciflua L. a species of potential for the tropics. Commonwealth Forestry Review, London, v. 63, n. 3, p. 207 - 216, 1984.

MENEGAZZO, M. L. Características morfológicas de celuloses branqueadas de Pinus e Eucalyptus em analisador óptico automático de fibras. 65f. Dissertação (Mestrado em Ciências Florestais) Faculdade de Ciências Agronômicas, Universidade Estadual Paulista. Botucatu, 2012.

MUNSEL, A. H. Munsel book of color: defining, explaining and illustrating the fundamental characteristis of color. Baltimore: Munsel Color Company, 1957. $70 \mathrm{p}$. 
OLIVEIRA, J. T. S. Propriedades físicas e mecânicas da madeira. In: OLIVEIRA, J. T. S.; FIEDLER, N. C.; NOGUEIRA, M. (Eds.). Tecnologias aplicadas ao setor madeireiro. Visconde do Rio Branco: Suprema Gráfica e Editora, 2007.p. 129 - 163.

OLIVEIRA, J. T. S.; HELLMEISTER, J. C.; TOMAZELLO FILHO, M. Variação do teor de umidade e da densidade básica na madeira de sete espécies de eucalipto. Revista Árvore, Viçosa, v. 29, n. 1, p.115 $127,2005$.

PIGG, K. B.; ICKERT-BOND, S. M.; WEN, J. Anatomically preserved Liquidambar (Altingiaceae) from the Middle Miocene of Yakima Canyon, Washingon State, USA, and its biogeographic implications. American Journal of Botany, St. Louis, v. 91, p. 499 - 509, 2003.

RAMALHO, R. S. O Uso de macerado no estudo anatômico de madeira. Viçosa: UFV, 1987. 4 p.

SANTOS, S. R. Influência da qualidade da madeira de híbridos de Eucaliptus grandis $\mathbf{x}$ Eucalyptus urophylla e do processo Kraft de polpação na qualidade da polpa branqueada. $160 \mathrm{f}$. Dissertação (Mestrado em Recursos Florestais) - Universidade de São Paulo, Piracicaba, 2005.

SILVA, M. G. Produtividade, idade e qualidade da madeira de Eucalyptus destinada à produção de polpa celulósica branqueada. $94 \mathrm{f}$. Dissertação (Mestrado em Tecnologia de Produtos Florestais) Universidade de São Paulo, Piracicaba, 2011.

SEBBENN, A. M.; ARANTES, F. C.; VILAS BÔAS, O.; FREITAS, M. L. M. Performance of 19-yearold provenance of Liquidambar styraciflua in Paraguaçu Paulista, São Paulo. Crop Breeding and Applied Biotechnology; Viçosa, v. 7, n. 4, p. 381 - 386, 2007.

SHIMIZU, J. Y. Liquidambar para produção de madeira no sul e sudeste do Brasil. Pesquisa Florestal Brasileira, Colombo, v. 50, n. 1, p. 127 - 138, 2005.

SHIMIZU, J. Y.; SPIR, I. H. Z. Produtividade de madeira de liquidambar (Liquidambar styraciflua L.) de diferentes procedências, em Quedas do Iguaçu, PR. Revista Árvore, Viçosa, v. 28, n. 4, p. 487 $491,2004$.

SOUSA, L. C. Caracterização da madeira de tração em Eucalyptus grandis e sua influência na produção de polpa celulósica. 77 f. Tese (Doutorado em Ciência Florestal) - Universidade Federal de Viçosa, 2004.

FLORESTA, Curitiba, PR, v. 45, n. 4, p. 723 - 734, out. / dez. 2015. 